\title{
Location of Partial Discharge Sources and Analysis of Signals in Chosen Power Oil Transformers by Means of Acoustic Emission Method
}

\author{
A. Olszewska and F. Witos* \\ Department of Optoelectronics, Silesian University of Technology \\ B. Krzywoustego 2, 44-100 Gliwice, Poland
}

\begin{abstract}
The original system useful for analysis of signals recorded during investigations of partial discharges within power oil transformers by means of acoustic method is presented. This method includes the basic and advanced analysis of recorded data. In the frame of basic analysis of data recorded signals undergo filtration in chosen frequency bands and next the analysis is made - in domain of time, frequency, time-frequency and discrimination threshold. In the frame of advanced analysis of data the amplitude distributions of acoustic emission signals and the acoustic emission descriptors (defined by the authors) are calculated in order to outline maps of acoustic emission descriptors on lateral walls of a transformer; it is a base for location of sources of partial discharges by means of the original method consisted in determination of advance degree of a signal. Results of this analysis, for signals recorded in two chosen transformers with identical construction (partial discharge occurred only within one of them), are presented in the paper. The source of partial discharge, situated within oil near transformer tank, was localized; the revision confirmed this result. Properties of recorded emission acoustic signals at chosen measuring points situated on the tank, in function of distance between the partial discharge source and measuring points, are presented.
\end{abstract}

PACS: 52.80.-s, 77.22.Jp, 84.70.+p, 43.40.Le, 43.60.Lq

\section{The original system of analysis of acoustic emission signals}

The original system of analysis of acoustic emission (AE) signals includes the basic and advanced analysis of recorded data, dedicated to location and description of partial discharges (PD) within power oil transformers by means of AE method. The basic analysis of recorded signals is made by means of $A E+J F T A$ program, written in LabView environment. The program contains the main level and detailed ones. In the first order, data from the set created during registration process should be read in. The next step comes down to determination of data for analysis, by means of selection of measuring channel and length of analyzed signal. Afterwards, one should pass to detailed levels and to define over there suitable parameters which control calculations. After determination of data and parameters the program is ready to realization of calculations. Calculations enable us to analyze signals in domain of time, frequency, timefrequency and discrimination threshold. Example calculation results obtained from the program for one signal in one measuring channel is presented in Fig. 1. These results are composed of: signal after filtration (for filtration there is used a band-pass filter of 5 . order) -

* corresponding author; e-mail: Franciszek.Witos@polsl.pl
Fig. 1a, phase-time characteristic - Fig. 1b, averaging phase characteristic - Fig. 1d, three-dimensional shorttime Fourier transform (STFT) spectrogram - Fig. 1c, three-dimensional STFT spectrogram projected to the plane of phase-frequency - Fig. 1e. AE+JFTA program is used also to calculations for a sequence of detailed levels determined in batch-file set for all sets in a chosen folder. It is useful for analysis of data recorded in the net of measuring points displaced on the tank of tested transformer [1].

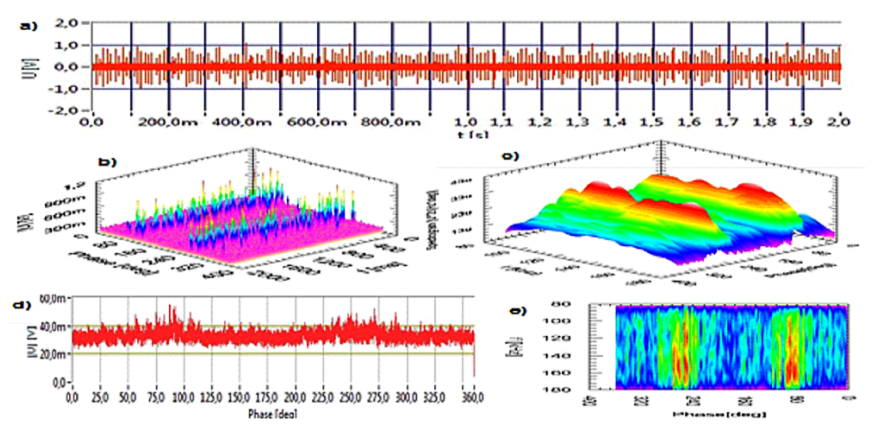

Fig. 1. Description of a signal coming from PD after filtration in the band of $80-180 \mathrm{kHz}$ : (a) signal, (b) phase-time characteristic, (d) averaging phase characteristic, (c) and (e) STFT spectrograms. 
In the frame of advanced analysis the following amplitude distributions are calculated: power of AE signals, counting rate. Amplitude distributions are the basis of the original advanced analysis of signals assigned to AE descriptors with acronyms: ADC (amplitude distribution of AE counts) and ADP (amplitude distribution of power of AE signal).

The way how descriptors are defined is presented in Fig. 2, in example of ADC descriptor. First, one should determine at amplitude distribution such a fragment which is conformed with discrimination threshold range: from $U_{\mathrm{d}}$ (minimum of derivative of distribution calculated towards threshold) up to $U_{\mathrm{g}}$ ( $90 \%$ of maximal value of recorded signal). This fragment of the curve is approximated by the straight line, whereas the descriptor connected with the distribution is equal to value of slope of this straight line.
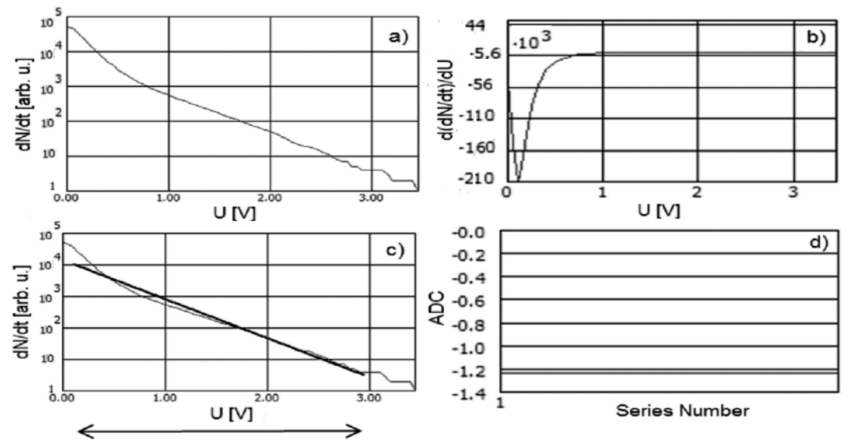

Fig. 2. Calculation of ADC descriptor: (a) amplitude distribution of counting rate, (b) derivative of amplitude distribution of counting rate, (c) amplitude distribution of counting rate with approximation curve and range of discrimination threshold $\left(U_{\mathrm{d}}\right.$ and $\left.U_{\mathrm{g}}\right),(\mathrm{d})$ ADC descriptor for amplitude distribution from Fig. 2a.

Descriptors defined in such a way consider physical features of investigated phenomenon, connected with propagation and thickness of interconnected layer (logarithmic scale of values in amplitude distributions). Descriptors are not based on values measured immediately and owing to elimination of principle limits of acoustic measuring methods - describe recorded AE signals and assign them the so-called degree of advance of AE signal. The degree of advance of AE signal is connected with the degree of advance of deformation process and considers regularity with which deformation process occurs in AE source, whereas AE signal is recorded at a measuring point. Descriptors take negative values - greater values of descriptor (more flat fragment of a curve) means a higher (more advanced) degree of AE signal. These properties of descriptors are presented in Fig. 3 for family AE signals recorded in one measuring channel and during measuring situations where tested object were energized by higher and higher voltage. Descriptors show development of deformation processes when the supply voltage increases [1-5].
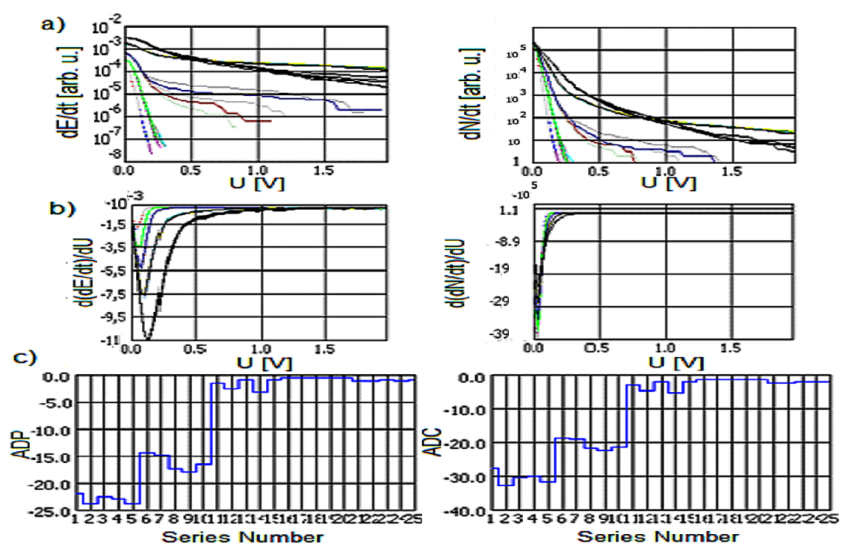

Fig. 3. Investigations of properties of amplitude distributions by means of descriptors: (a) amplitude distributions, (b) derivative of amplitude distributions, (c) AE descriptor for amplitude distributions from Fig. 3a.

Calculated descriptor values compose input data for determination of AE descriptor maps on lateral walls of transformer tanks. These maps are made by means of kriging method, basing on all data. Isolines in the maps connect points where calculated values of descriptors are the same. AE descriptor maps locate areas on lateral walls of transformers where AE signals reach, coming from partial discharges. Designation of areas of maps, in which the descriptors achieve local maxima is defined by authors as location by means of method of advancedd degree of signals. The maps - in connection with results of basic analysis and information on construction of tested transformer - are the basis for location of PD sources within tested power oil transformer.

\section{Tested objects}

Investigations have been made in two power oil transformers (signed as T-1 and T-2) with rated power of $25 \mathrm{MVA}$ and rated voltage $110 \mathrm{kV}$; they were realized by means of the origin AE measuring system DEMA-COMP [1-5] equipped with R6 sensors produced by Physical Acoustic Corporation. The transfer band of AE measuring system DEMA-COMP is from $20 \mathrm{kHz}$ to $500 \mathrm{kHz}$ and coincides with the analysis recommended by other studies [6-10].

Both transformers are of the same construction but they are differing in operational parameters. Results of the same last testing of the oil come from T-2 transformer, made by means of chromatographic analysis (DGA), point out existence of partial discharges within this transformer. Level of gases within T-1 transformer is contained in the range acceptable by appropriate standards.

Measurements were carried out at power station during operation of transformers. Signals were recorded at chosen and accessible measuring points on lateral surfaces of the tank. Duration of each signal was 2 s (100 periods of 
the supply voltage). Signals were recorded several times at each measuring point.

\section{Maps of descriptors}

In the frame of analysis, recorded signals were filtrated in the first order in the band of $20-100 \mathrm{kHz}$ and next the following quantities have been calculated: basic characteristics, amplitude distributions and AE descriptors with ADC acronym [1-5]. AE descriptors compose input data for tracing maps of descriptors on lateral walls of tanks of tested transformers. These maps are created by means of cringing method. Isolines on the maps connect the points where calculated values of descriptors are the same.

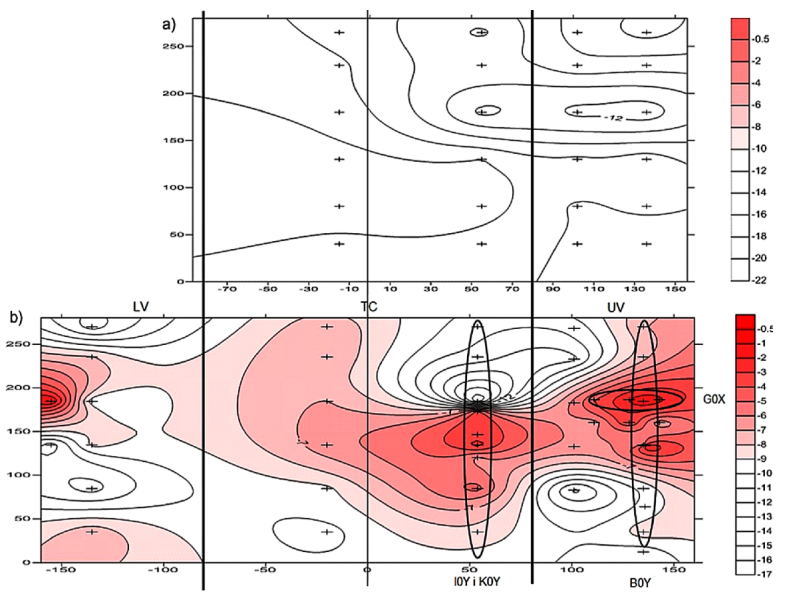

Fig. 4. Map of ADC descriptor calculated after the analysis of detected AE signals within the band of 20-100 kHz for T-1 transformer (a) and T-2 transformer (2). Dimensions of the tank $(X, Y)$ in $\mathrm{cm}: X-$ running position along the tank, 0 - center of the tap changer, positive values of $X$ - part of the tank from the side of the upper voltage, negative values of $X$ part of the tank from the side of the low voltage, $Y$ running height on the tank, +- measuring points.

Elaborated maps of ADC descriptors for analyzed frequency band are presented in Fig. 4a (for T-1 transformer) and in Fig. 4b (for T-2 one). $X Y$ plane on these maps is developed lateral plane of the tank. Coordinates of points at this plane, given in $\mathrm{cm}$, are determined in the following way: values of $Y$ coordinates are calculated from the bottom of the tank, the value of $X$ coordinate is equal to zero for the center of the tap changer, values of $X$ coordinates increase in direction of the side of the upper voltage, values of $X$ coordinates decrease in direction of the side of the low voltage; extreme values of $X$ coordinates describe points opposite to ones which describe the center of the tap changer. Darkest colors on the map indicate places with greater values of descriptor, and - in the same - with greater degree of advance of AE signal, recorded at a measuring point.

\section{Location of PD sources and analysis of AE signals}

Every values of ADC descriptors for signals recorded in T-1 transformer (Fig. 5a) are small which signifies lack of partial discharges within this transformer. Example description of AE signal for T-1 transformer is presented in Fig. 5. Results of analyses prove that these characteristics describe an acoustic noise of working transformer.

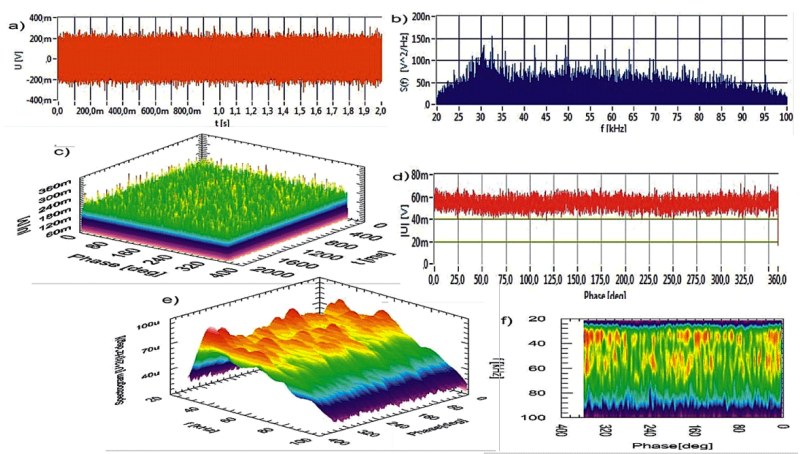

Fig. 5. Introductory description of the AE signal recorded at selected measuring point of $\mathrm{T}-1$ transformer: (a) impulse, (b) frequency characteristic, (c) phase-time characteristic, (d) averaging phase characteristic, (e), (f) averaging STFT spectrograms, $\mathrm{ADC}=-20.63$ (file TR2M06(1).BIN).

In the case of T-2 transformer (Fig. 5b), ADC descriptor takes considerably greater values which prove that deformational (degradation) processes within this transformer are more advanced. Three areas with high degree advance of AE signals were located in:

(a) area - the side of the upper voltage with coordinates: $X=130-150 \mathrm{~cm}, Y=180-190 \mathrm{~cm}$,

(b) area - the tap changer,

(c) area - the side of the low voltage with coordinates: $X=145-155 \mathrm{~cm}, Y=180-190 \mathrm{~cm}$.

When areas on lateral walls of transformer (where AE signals from PD reach) are located one should analyze basic characteristics of signals coming from chosen measuring points in order to locate PD sources and analyze propagation of signals from the source.

Analysis of properties of AE signals has been made for chosen measuring points, situated in measuring columns/ rows of T-2 transformer which are located in areas indicated above as (a) or (b):

(a1) measuring points in (a) area in the column B: B01, B02, B03, B04, B05 and B06,

(a2) measuring points in (a) area in the row G: G01, G02 and G03,

(b) measuring points in (b) area: I01-I06 and K01-K03.

Characteristics belonging to the basic description of chosen signals recorded in the area (a) are presented in Figs. 6 and 7. The signal recorded at B04 point occurs regularly twice over each period of the supply voltage. It 
has very great amplitudes and dominant frequency band of $40-60 \mathrm{kHz}$. There are two good located areas at phase-frequency characteristic of a signal (Fig. 7e). Results of analyses prove that it is description of the signal coming from the PD source. Basic characteristics of a signal, recorded at the measuring point $\mathrm{B} 03$, situated $50 \mathrm{~cm}$ below the source, have a similar character. The signal occurs twice over a period of the supply voltage. Frequency band is displaced in the side of lower frequencies; it is connected with damping of a signal in longer way of its propagation. Such a signal has smaller amplitudes, similar phases and it is worse located; wider phase band is observed.

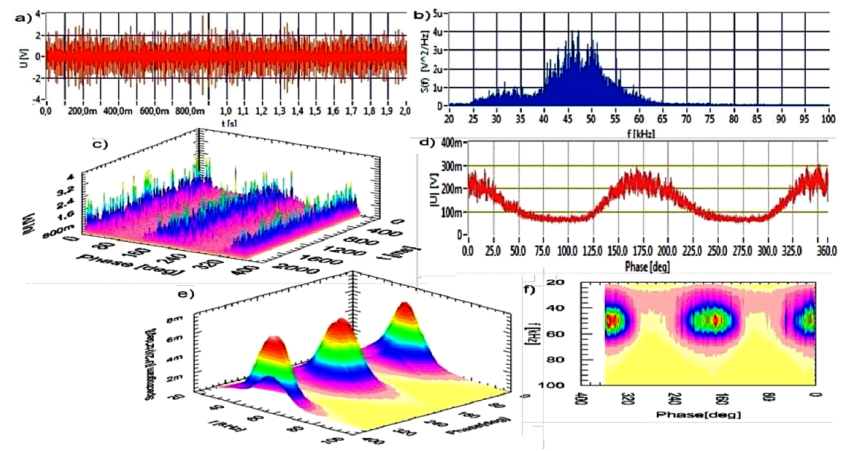

Fig. 6. Introductory description of the AE signal recorded at selected measuring point of T-2 transformer: (a) impulse, (b) frequency characteristic, (c) phase-time characteristic, (d) averaging phase characteristic, (e), (f) averaging STFT spectrograms, $\mathrm{ADC}=-1.28$ (file T1B04(2).BIN).

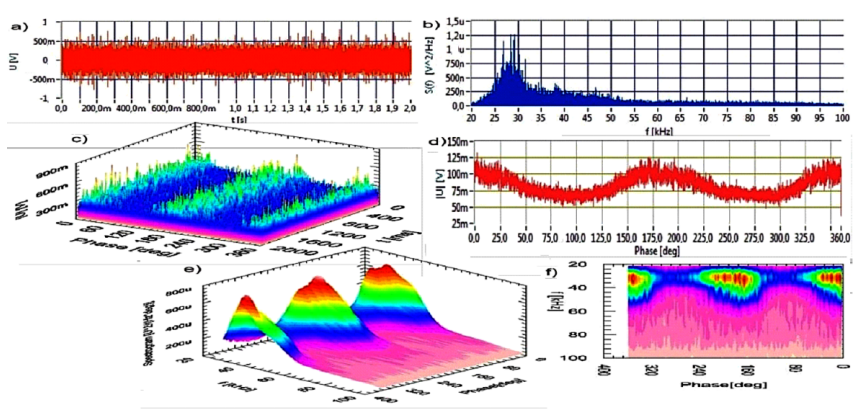

Fig. 7. Introductory description of the AE signal recorded at selected measuring point of $\mathrm{T}-2$ transformer: (a) impulse, (b) frequency characteristic, (c) phase-time characteristic, (d) averaging phase characteristic, (e), (f) averaging STFT spectrograms, $\mathrm{ADC}=-5.81$ (file T1B03(2).BIN).

Averaging phase characteristics of signals recorded at measuring points BOY $(Y=1,2, \ldots, 6)$ are presented in Fig. 8. Positions of maxima in these characteristics are calculated and these results are presented in Table. Changes of position of such a maximum, calculated towards position of maximum in the characteristic for B04 point, are visible. An essence of these changes is as

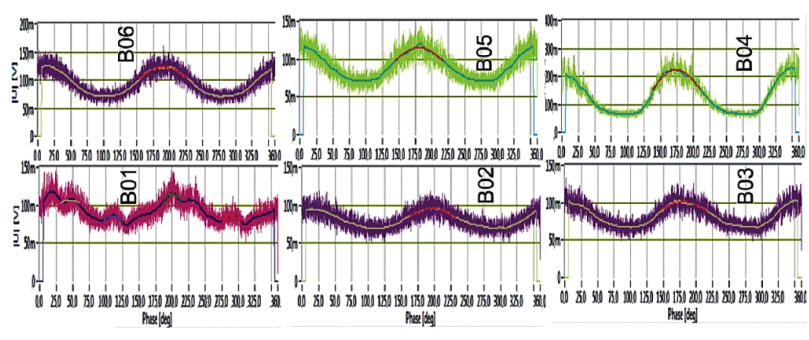

Fig. 8. Averaging phase characteristics of the AE signals recorded at selected measuring points of T-2 transformer (area (a), measuring points (a1): $\mathrm{B} 0 Y, Y=$ $1,2,3,4,5$ and 6$)$. Points displaced according to position on wall of the tank from the top (B06) to the bottom (B01).

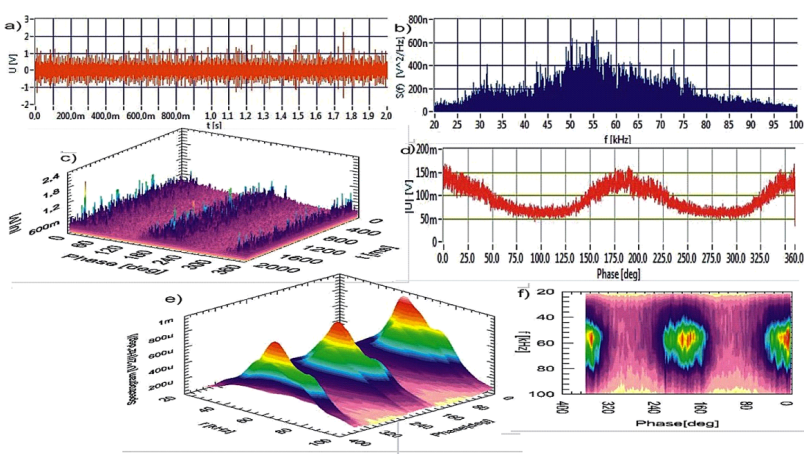

Fig. 9. Introductory description of the AE signal recorded at selected measuring point of $\mathrm{T}-2$ transformer: (a) impulse, (b) frequency characteristic, (c) phase-time characteristic, (d) averaging phase characteristic, (e), (f) averaging STFT spectrograms, $\mathrm{ADC}=-2.59$ (file T1I03(2).BIN).

follows: maxima at characteristics for signals recorded at particular measuring points occur for greater phase value while these points are carried away from B04 point. If velocity of longitudinal wave within the oil is about $1200 \mathrm{~m} / \mathrm{s}$ [9] then - converting difference of phases between the source and particular measuring points in B0Y ((a1) measuring points) into difference of propagation ways - one should obtain results presented in Table. Differences of propagation ways between signals recorded at measuring points B04 and B03 as well as B04 and B05 are $42 \mathrm{~cm}$ and $47 \mathrm{~cm}$, accordingly. It needs to add that distance between these points on the tank is $0.5 \mathrm{~m}$. Additionally it is known that PD source located in vicinity of measuring point B04 is situated near the tank of transformer. That proved revision of T-2 transformer. It results from that the signal coming from a source propagates in analyzed area without greater obstacles and occurs at other measuring points with delay according to followed way.

Measuring points (a2) are situated in nearest surrounding of localized PD source. They have similar character as characteristics obtained for the source. Measuring point G01 is situated $8 \mathrm{~cm}$ from signal source so the band of frequency and phase for signal recorded at this point 


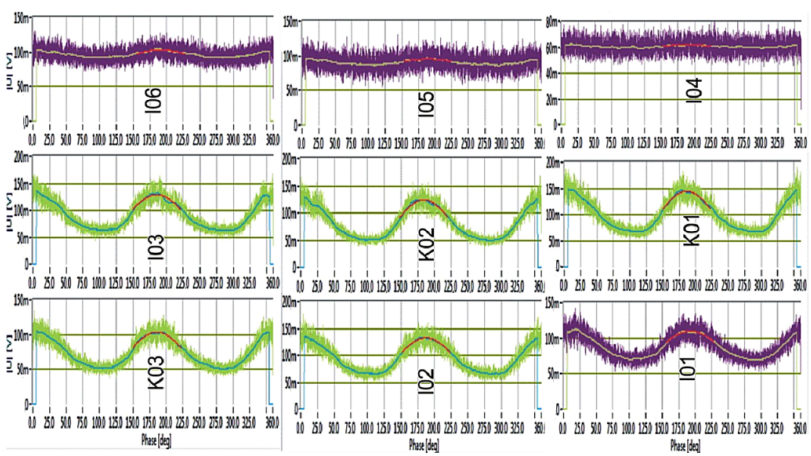

Fig. 10. Averaging phase characteristics of the AE signals recorded at selected measuring points of T-2 transformer (files T1I0X and T1K0X). Points displaced according to position on the wall of transformer from the top (I06) to the bottom (I01), points K01, K02, I03, K03 placed on the flap of tap changer.

are similar to previous characteristics (Fig. 7). For this group of signals, declination of the phase while points are carried away from the source is observed, too. Phase values converted into the way of propagation for signal recorded in this area are collected in Table. Points G02, B04 and G01 are situated at small distance of ones from the others, so the signal coming from the source to particular points attaint them with small phase delay. Results of analysis of phase shift for such a group of signals prove that PD source is situated between points B04 and G01.

The last groups of signals are AE signals recorded at measuring points (b): I01-I06 and K01-K03 (in (b) area - the tap changer). Characteristics belonging to the basic description of chosen signals recorded in this area are presented in Figs. 9 and 10. These signals are periodical, occur twice in each period of the supply voltage. There are two areas, well located at phase-frequency characteristics. As before, differences of phases for particular signals at averaging phase characteristics of modulus of signals recorded at measuring points belonging to (b) area (Fig. 10) are visible; signals occur later in the phase while the point is more distant from the source. As can be seen in the last column of Table, the smallest calculated distance value is $69 \mathrm{~cm}$, which is consistent with expectations as the PD source is inside the transformer near the point B04. The area indicated as (b) is complex, which means that the signals generated in the source of $\mathrm{PD}$ reach only certain measuring points located on the tank. Generally, this image is also evidence of the complexity of the propagation path of signals radiated by the $\mathrm{PD}$ source. It is also worth compare the frequency characteristics in Fig. 7 and 8 with those in Figs. 9 and 10. Characteristics in Fig. 9 and 10 describe the signals that have traveled longer distances and at frequency characteristics this is seen as a change in relations between the two peaks: a maximum lying in the lower frequency band decreases more slowly than the maximum lying in the higher frequency band.

TABLE

Maximum values of phases with converting into difference of propagation ways for signals recorded in (a) and (b) areas in successive measuring points.

\begin{tabular}{c|c|c|c|c|c|c|c|c}
\hline \hline \multicolumn{3}{c|}{ (a1) from (a) area } & \multicolumn{3}{c|}{ (a2) from (a) area } & \multicolumn{3}{c}{ (b) from (b) area } \\
\hline $\begin{array}{c}\text { Meas. } \\
\text { points }\end{array}$ & $\phi_{\max }\left[^{\circ}\right]$ & $d[\mathrm{~m}]$ & $\begin{array}{c}\text { Meas. } \\
\text { points }\end{array}$ & $\phi_{\max }\left[^{\circ}\right]$ & $d[\mathrm{~m}]$ & $\begin{array}{c}\text { Meas. } \\
\text { points }\end{array}$ & $\phi_{\max }\left[^{\circ}\right]$ & $d[\mathrm{~m}]$ \\
\hline B06 & 190.684 & 1.13 & G03 & 179.195 & 0.37 & I06 & 191.176 & 1.17 \\
B05 & 180.689 & 0.47 & G02 & 174.314 & 0.05 & I05 & 189.201 & 1.04 \\
B04 & 173.654 & - & B04 & 173.654 & - & I04 & 188.305 & 0.98 \\
B03 & 180.005 & 0.42 & G01 & 173.137 & 0.04 & K01 & 186.429 & 0.85 \\
B02 & 196.354 & 1.51 & & & & K02 & 184.062 & 0.69 \\
B01 & 200.413 & 1.78 & & & & I03 & 185.156 & 0.77 \\
& & & & & & K03 & 185.872 & 0.81 \\
& & & & & & I02 & 187.384 & 0.92 \\
\end{tabular}

\section{Recapitulation}

The system used to analysis of AE signals, recorded within power oil transformers, is presented. Maps of descriptors together with results of basic analysis and information about structure of tested power oil transformer enable us to locate PD sources within such equipment.

Testing of T-1 transformer proved that there are only acoustic noises within this object but not partial dis- charges. Owing to analysis of AE signals within T-2 transformer an advanced PD source, placed in vicinity of measuring point B04, has been identified (horizontally $140-150 \mathrm{~cm}$, vertically $180-190 \mathrm{~cm}$ ). That was proved by revision of T-2 transformer (conductors connecting $\mathrm{HV}$ lead and the tap changer lied too close of the tank).

Analysis of properties of AE signals recorded in three areas of T-2 transformer brought to location of the PD 
source and proved that - in the case of the source is localized in oil near the tank - phase displacements between particular signals correspond with differences of propagation ways of signals from the source to the measuring point.

Subsequent investigations aimed at elaboration of the method useful to three-dimensional location of PD sources within power oil transformers are intended.

\section{Acknowledgments}

Investigations have been financed in the frame of the project N N505 466538 of Ministry of Science and Higher Education/National Center for Science.

\section{References}

[1] F. Witos, Investigation of Partial Discharges by Means of Acoustic Emission Method and Electric Method, SUT, Gliwice 2008 (in Polish).

[2] F. Witos, Z. Gacek, A. Opilski, Arch. Acoust. 27, 65 (2002).
[3] F. Witos, Z. Gacek, Europ. Phys. J. ST 154, 239 (2008).

[4] F. Witos, Z. Gacek, Acta Phys. Pol. A 116, 422 (2009).

[5] F. Witos, A. Olszewska, Acta Phys. Pol. A 118, 1267 (2010).

[6] Z. Deheng, T. Kexiong, T. Xianche, in: Properties and Application of Dielectric Materials, 2nd Int. Conf. Record, Ed. W. Huang, Univ. Bejing, Bejing 1988 , p. 614

[7] S. Markalous, S. Tenbohlen, K. Feser, IEEE Trans. Diel. Electr. Insulat. (ITDEIS) 15, 1576 (2008).

[8] R. Witula, D. Slota, J. Math. Anal. Appl. 324, 321 (2006).

[9] High-Voltage On-Site Testing with Partial Discharge Measurement; Working Group D1.33, Task Force 05, June 2012, ISBN: 978-2-85873-194-7.

[10] T. Boczar, S. Borucki, A. Cichon, M. Lorenc, Acta Phys. Pol. A 114, A-21 (2008).

[11] E. Howells, E.T. Norton, IEEE Trans. Power Apparat. Syst. PAS-103, 1111 (1984). 